\title{
Gene panels and primers for next generation sequencing studies on neurodegenerative disorders
}

\author{
Vo Van Giau ${ }^{1, *}$, Seong Soo A. An ${ }^{1, *}$, Eva Bagyinszky ${ }^{1}$ \& SangYun Kim ${ }^{2}$
}

(C) The Korean Society of Toxicogenomics and Toxicoproteomics and Springer 2015

Erratum to: Mol Cell Toxicol (2015) 11:89-143

DOI 1.1007/s13273-015-0011-9

In this article, acknowledgements was omitted.
Acknowledgements This work was supported by the grants from by Korea National Research Foundation (NRF2010-0024004) and the Korea Health Technology R\&D Project (HI14C3331) through the Korea Health Industry Development Institute (KHIDI), Korea Ministry of Health \& Welfare.

The online version of the original article can be found under doi: 1.1007/s13273-015-0011-9.

${ }^{1}$ Department of Bionano Technology, Gachon Bionano Research Institute, Gachon University, 1342 Seongnam-daero, Sujeong-gu, Seongnam-si, Gyeonggi-do 461-701, Korea

${ }^{2}$ Department of Neurology, Seoul National University College of

Medicine \& Neurocognitive Behavior Center, Seoul National University

Bundang Hospital, 300 Gumidong, Bundang-gu, Seongnam-si,

Gyeonggi-do, Korea

*These authors contributed equally to this work.

Correspondence and requests for materials should be addressed to

E. Bagyinszky ( $\varangle$ navigator120@gmail.com) \&

S.Y.Kim( $\square$ neuroksy@snu.ac.kr) 